\title{
Transcriptome-wide analysis of immune-responsive microRNAs against poly (I:C) challenge in Branchiostoma belcheri by deep sequencing and bioinformatics
}

\author{
Qi-Lin Zhang ${ }^{1,2, *}$, Qian-Hua Zhu ${ }^{3, *}$, Feng Zhang ${ }^{1,2}$, Bin $X_{u^{1,2}, \text { Xiu-Qiang Wang }}^{1,2}$ and \\ Jun-Yuan Chen ${ }^{1,2}$ \\ ${ }^{1}$ State Key Laboratory of Pharmaceutical Biotechnology, School of Life Science, Nanjing University, Nanjing, China \\ ${ }^{2}$ LPS, Nanjing Institute of Geology and Paleontology, Nanjing, China \\ ${ }^{3}$ Beijing Genomics Institute, Shenzhen, China \\ * These authors contributed equally to this work \\ Correspondence to: Jun-Yuan Chen, email: chenjy@nju.edu.cn
}

Qi-Lin Zhang, email: qlzhang11@|zu.edu.cn

Keywords: Branchiostoma belcheri, miRNA, poly (I:C), deep sequencing, Immunology and Microbiology Section, Immune response, Immunity

Received: July 13, $2017 \quad$ Accepted: August 04, $2017 \quad$ Published: August 28, 2017

Copyright: Zhang et al. This is an open-access article distributed under the terms of the Creative Commons Attribution License 3.0 (CC BY 3.0), which permits unrestricted use, distribution, and reproduction in any medium, provided the original author and source are credited.

\section{ABSTRACT}

Amphioxus is a key experimental animal for studying the evolution of vertebrate immune system. However, we still do not know about the roles of microRNAs (miRNAs) under viral stress in amphioxus. In this study, we sequenced six small RNA libraries (three biological replicates were included in the treatments challenged by the viral mimic, poly (I:C) (pIC) and control groups, respectively) from Branchiostoma belcheri. A total of 151 known miRNAs, 197 new miRNAs (named novel_mir, including nine conserved miRNAs) were identified by deep sequencing from the six libraries. We primarily focused on differentially expressed miRNAs (DEMs) after pIC challenge. Next, we screened a total of 77 DEMs, including 27 down- and 50 up-regulated DEMs in response to pIC challenge. Furthermore, we used real-time quantitative PCR (qRTPCR) to verify the expression levels of 10 randomly selected DEMs. Target genes likely regulated by DEMs were predicted, and functional enrichment analyses of these targets were performed using bioinformatics approach. MiRNA targets of DEMs are primarily involved in immune response, diseases, cancer and regulation process, and could be largely linked to 14 immune-related signaling pathways, including NF-kappa B, NOD-like receptor, RIG-I-like receptor and endocytosis. The present study for the first time explores key regulatory roles of miRNAs in the innate antiviral immune response in amphioxus, and will provide insight into the molecular basis of antiviral immunity and evolution of immune-related miRNAs.

\section{INTRODUCTION}

Sister subphyla, the Cephalochordata, Urochorda, and Vertebrata, is all the three members of phylum Chordata [1]. Amphioxus is the living representative of Cephalochordata, and is considered to be the last common ancestor of chordates owing to its proximal morphological and genomic evidence with the ancient chordate group $[2,3]$. This group usually burrows shallow in temperate and tropical seas with growth fuel of microorganisms [4]. Amphioxus must thus face acute invading microorganisms, which facilitates adaptive evolution of its innate immune system [4]. Indeed, many experiments reported that amphioxus is susceptible to certain bacteria, bacterial and viral mimics, such as lipopolysaccharide (LPS) and poly (I:C) (pIC; a double-stranded RNA viral mimic) [5-7]. Many studies have found acute changes in expression of genes/microRNAs (miRNAs) upon immune challenge in amphioxus [8-10]. Thus, amphioxus can serve as a model for investigating primitive innate immune responses, signaling pathways and exploring the evolution of key immune-related molecules. 
miRNAs, a class of endogenous non-coding small RNAs, are transcribed initially by RNA polymerase II, which are then processed by Drosha and Dicer-1 enzymes to form miRNA: star-miRNA duplexes[11]. Following cleavage by Dicer, more stable miRNAs (approximately $22 \mathrm{nt})$ preferentially incorporate into an RNA-induced silencing complex (RISC). Next, miRNAs guide the RISC to target complementary sequences in the 3' UTR (untranslated regions) of mRNAs to negatively regulate gene expression, thereby blocking the translation of target transcripts or degrading mRNA [12-14]. Many previous studies have recognized that miRNA molecules are important regulators of immune, inflammatory and disease response in animals [15-17]. For example, some miRNAs, such as miR-155, miR-223, miR-181a, miR-17-92 cluster and miR-146 family mediate expression of target genes associated with immune cell development and function in mammal hematopoiesis [18]. Previous studies speculated that miR-146a, miR-155 and miR-9 suppressed the acute responses following activation of innate immune system by negatively regulating the expression of genes involving receptor-induced signaling pathways in vertebrates [19]. $\mathrm{Lu}$ et al. found that the absolute decrease in abundance of many microRNAs, including the let-7 family, could significantly promote oncogenes is in human cancer [20]. Recently, Jin et al. identified 14 differentially expressed miRNAs (DEMs) in Branchiostoma belcheri infected with Vibrio parahemolyticus when compared with the control using microarray profiling [10]. Liao et al. detected 56 DEMs in gills of B.belcheri after LPS challenge using microarray technology [9]. Yang et al. found that bbe-miR-7, bbe-miR-4868a, bbe-miR-2065, bbe-miR-34b and miR-92d were differently expressed in $B$. belcheri upon infection with both Vibrio anguillarum and Staphylococcus aureus [21]. These studies focus on roles of miRNAs in immune response of amphioxus only to challenges with bacteria or bacterial mimics. However, no studies have been done yet to analyze the function of miRNAs in the antiviral immune defense in amphioxus.

Currently, with the development of highthroughput miRNA deep sequencing technologies, wholetranscriptome/genome identification and expression analysis of miRNAs has been widely conducted to massively and efficiently screen for DEMs involving immune responses and explore their roles in vertebrate immunity [22, 23]. Additionally, in previous immunerelated studies in amphioxus, hybridization-based microarray analysis has been the predominant method for detecting acute immune responses of miRNAs to challenges with pathogens and their mimics [9, 10]. However, recently, Jin et al. systematically identified expression of miRNAs under bacterial infection using miRNA Solexa deep sequencing, thus providing a full suite of candidate probes for subsequent preparation of microarrays to analyze differential expression of miRNAs [10]. Evidently, when compared with hybridization- based technologies such as microarrays, miRNA deep sequencing can identify miRNAs expressed even at low abundance and reliably read sequences of miRNAs among different samples [24]. Thus, deep sequencing has been a powerful approach for analysis of transcriptome-wide immune-responsive miRNAs in amphioxus species with sequenced genomes, including Branchiostoma floridae and $B$. belcheri $[2,25]$.

In the present study, to investigate the miRNA expression changes in antiviral immunity and to provide insights into the regulated roles of miRNA in immune signaling pathways in amphioxus, we performed transcriptome-wide analysis of immune-responsive microRNAs under pIC challenge in B.belcheri using deep sequencing and bioinformatics methods. Known, conserved and novel DEMs were identified using the bioinformatics approach. We then performed quantitative real-time PCR (qRT-PCR) analysis for a subset of randomly selected DEMs to validate the sequencing data. To further understand the functions of these DEMs, the corresponding target genes were predicted and then analyzed using enriched Gene Ontology (GO) terms and Kyoto Encyclopedia of Genes and Genomes (KEGG) pathways. These findings should be helpful for better understanding the regulatory roles of miRNAs in antiviral immune response of $B$. belcheri.

\section{RESULTS}

\section{Analysis of small non-coding RNAs}

To identify immune-responsive miRNAs upon pIC challenge in B.belcheri, six small non-coding RNAs (sRNAs) libraries were sequenced from treated or control whole bodies of adult B.belcheri (three biological replicates for each of the treatment and control), generating 28.9, 29.6, 30.1 million (control groups) and 31.3, 29.9, 30.1 million (treated groups) raw tags. After performing quality control, 28.2, 28.7, 29.2 million clean tags were obtained from control samples, and corresponded to $97.63 \%, 97.13 \%$ and $97.01 \%$ of the total raw tags respectively; we obtained 30.0, 28.8, 29.2 million clean tags from treated samples, constituting 95.74\%, 96.51\% and $97.05 \%$ of the total raw tags respectively. The ratios between total mapped tags to the B.belcheri genome and clean tags ranged from $78.82 \%$ to $83.74 \%$ (Supplementary Table 1). Based on the transcripts per million (TPM) values of all detected sRNAs, Pearson's correlation values were calculated for pairwise comparisons among the six libraries. The values were $>0.80$ for comparisons among the three biological replicates from similar treatments, i.e., among the negative controls or among treated groups (Figure 1A), indicating that the experimental approaches in this study were reliable. 
The length distribution of clean tags in the six libraries was calculated, and sizes of sRNA were found to range from 17 to 33nt (Supplementary Figure 1). In all libraries, the most abundant length of sRNA was 22 $\mathrm{nt}$, followed by $23 \mathrm{nt}$ and $21 \mathrm{nt}$, which is consistent with the normal length of miRNAs in B.belcheri [10] and other animals, including Larimichthys crocea [22] and Bombyx mori [24]. The results showed that the percentage of miRNA tags increased from $\sim 30 \%$ in negative control groups to $\sim 40 \%$ in pIC-treated groups (Supplementary Table 1), indicating that miRNA molecules play a key regulatory role in antiviral immune response of B.belcheri to pIC challenge. We detected 162, 165 and 164 known miRNAs in the three control group replicates, of which 132 known miRNAs were shared among all three libraries, and were considered to be the known miRNA set of negative control for further analysis. Similarly, 169, 168 and 168 known miRNAs were found in the treated groups, of which 136 were common and considered as known miRNAs. By getting union of known miRNAs between the control and treated groups, we obtained a total of 151 known miRNAs for downstream screening of DEMs. Based on the total expression level (average TPM value of treatment plus that of control), we found that let-7, mir10 , mir-100 were the top three among highly expressed miRNA families, while members of the mir-10 family were the most abundant in the list of top 30 miRNAs (Supplementary Table 2).

\section{Identification of conserved and novel miRNAs in B. belcheri}

We found 211, 222 and 215 novel miRNA sequences in three biological replicates of control groups, while 217 , 220 and 214 novel miRNA sequences were obtained in the treated groups (Supplementary Table 1). By comparing the biological replicates, a total of 147 and 152 novel miRNA were shared within the control and treated groups respectively. Finally, we identified a total of 197 novel miRNA sequences by getting union for control and treated groups, which were analyzed further for DEMs. Novelmir51, novel-mir2, novel-mir108 were the top three novel miRNAs with high expression levels (Supplementary Table 3). Specifically, novel-mir51 showed the most abundant expression, which far exceeded that of other novel miRNAs, and was predicted to be a regulatory molecule of target genes encoding ribosomal protein S10 (S10). Furthermore, to investigate the conservation of these 197 novel miRNAs among animals, we identified nine conserved miRNAs belonging to eight families (Table 1). The homologs of the other novel miRNAs were
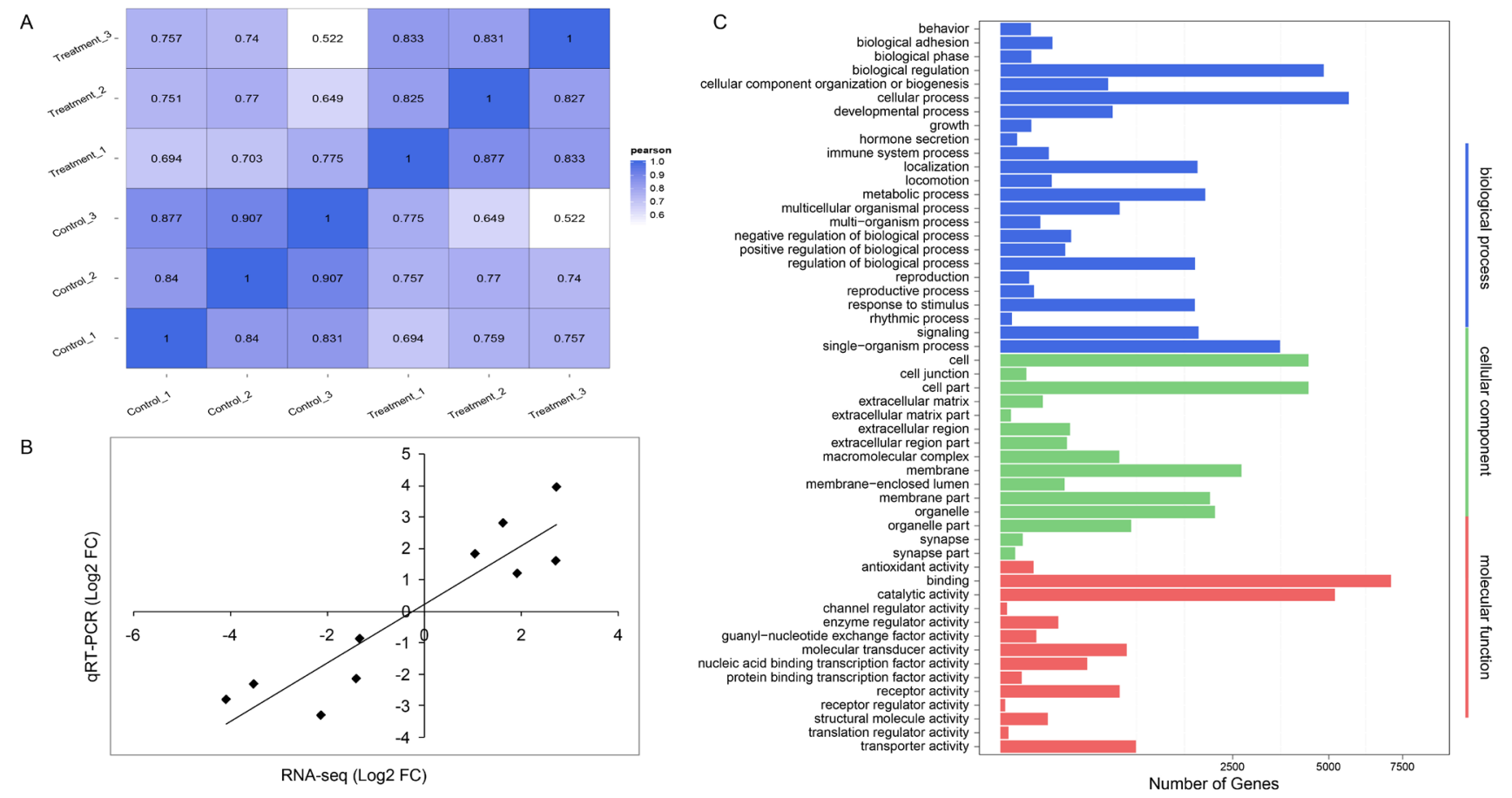

Figure 1: Reliability of deep-sequencing data and functional enrichment analysis. A. Heatmap of Pearson correlation coefficient among different samples. The maximum and minimum values are marked by blue and white respectively. A higher value indicates that the two samples are more similar. B. Correlation of Log2 (fold changes) in miRNA expression between deep sequencing and qRT-PCR results. The relative expression fold change between the control and treatment groups is indicated by black dots. C. GO terms enriched among target genes of differently expressed miRNAs. The x-axis indicates the number of target genes, and the y-axis shows the GO terms. All terms are classified into three subcategories marked by different colors. 
Table 1: Nine potential conserved Branchiostoma belcheri miRNAs newly identified by deep RNA-seq.

\begin{tabular}{|l|c|l|}
\hline \multicolumn{1}{|c|}{ Mature sequences (5'- 3') } & miRNA id & \multicolumn{1}{c|}{ Homologous miRNAs } \\
\hline acgcguuuaugucugcgccugu & novel_mir2 & bfl-miR-4862-5p \\
\hline ucgcauugacgucagcgccggc & novel_mir3 & bfl-miR-4856b-5p \\
\hline uagcuggcuggcgaagaaaug & novel_mir14 & bfl-miR-4900a \\
\hline uagcuggcuggcgaagaaaagc & novel_mir24 & bfl-miR-4900a \\
\hline aucuggggcaauuaagguuacga & novel_mir26 & bfl-miR-4894 \\
\hline uuuggcacugguacuuuggagu & novel_mir65 & bfl-miR-4871-3p \\
\hline gauguuuguacugucugucuguu & novel_mir108 & bfl-miR-4870 \\
\hline caagcucgugucuaugguucu & novel_mir136 & bfl-miR-100-3p \\
\hline ugagguaguagguuguagg & novel_mir152 & $\begin{array}{l}\text { hsa-let-7c-5p, mmu-let-7c-5p, rno-let-7c-5p, gga-let-7c-5p, dre-let-7c-5p, } \\
\text { ssc-let-7c, xtr-let-7c, etc }\end{array}$ \\
\hline
\end{tabular}

Bfl indicates Branchiostoma floridae; hsa, Homo sapiens; mmu, Mus musculus; rno, Rattus norvegicus; gga, Gallus gallus; dre, Danio rerio; ssc, Sus scrofa; xtr, Xenopus tropicalis.

not found, suggesting that they were not conserved in other animals and may potentially be $B$. belcheri-specific. Moreover, among the conserved miRNAs, sequences of five miRNAs (novel_mir3, novel_mir14, novel_mir24, novel_mir65, novel_mir108) have been reported to be involved in the immune response of $B$. belcheri to $V$. parahemolyticus infection [10]. Except for novel_mir152, eight out of a total of nine conserved miRNAs exist only in B. belcheri and B. floridae, indicating that they are novel amphioxus-specific miRNAs.

\section{Identification and $q R T-P C R$ validation of immune-responsive miRNAs}

We obtained 151 known and 197 novel (including nine conserved) miRNAs that were detected in at least one of the control and experimental groups. These were considered as candidates for further differential expression analysis. A total of 77 miRNAs, including 33 known and 44 novel (including two conserved) miRNAs, were identified to be differentially expressed in immune response to $\mathrm{pIC}$ challenge, of which 50 and 27 miRNAs were detected to be up- and down-regulated, respectively (Table 2). Among the DEMs, novel-mir27 was the most up-regulated, followed by novel-mir183 and novelmir121. These three novel miRNAs were predicted to regulate target genes encoding lipoxygenase 2 (LOX2), retinoic acid receptor (RAR), and putative oxidoreductase GLYR1 respectively by bioinformatics. Conversely, the top three DEMs that were most down-regulated after pIC challenge were novel-mir138, novel-mir158 and novelmir140. These were predicted to regulate genes encoding heat shock $70 \mathrm{kD}$ protein (HSP70), nucleotide-binding domain, leucine rich containing (NLR) protein family, and complement $\mathrm{C} 1 \mathrm{q}$-like protein $(\mathrm{C} 1 \mathrm{qL})$ respectively. Among known DEMs, the greatest up-regulation in expression was observed for bbe-miR-31-3p and bbe-miR-4856a-5p, which exhibited more than 3 -fold increase in abundance when compared with the control groups. Conversely, bbe-miR-2070-5p and bbe-miR-252a-5p were the most down-regulated (more than 4-fold changes) miRNAs after pIC challenge. The fold changes of 10 randomly selected DEMs between the deep sequencing and the qRT-PCR data were found to be significantly positively correlated (Pearson's correlation coefficient $=0.821$, two-side $P$-value $<0.01$ ) based on the linear regression analysis method (Figure 1B), indicating the accuracy of the miRNA deep sequencing method.

\section{Prediction of target genes and functional enrichment of immune-responsive miRNAs}

We used two prediction methods to predict the target genes of all DEMs. These putative target genes were then assigned to the three standard subcategories of "Biological Process", "Cellular Component" and "Molecular Function" in GO enrichment analysis (Figure 1C). In the "Biological Process" subcategory, 24 terms were enriched with the most abundant genes being involved in cellular processes, followed by biological regulation, and singleorganism process. Notably, three terms directly related to immune response, including immune system process, response to stimulus and signaling, were also detected. In the "Cellular Component" subcategory, 15 terms were assigned and the most abundant genes were related to cell, cell part and membrane. Lastly, 14 terms were assigned into "Molecular Function", of which binding and catalytic activity had the most abundant genes.

Furthermore, the target genes of all DEMs were also classified into 42 KEGG pathways (Supplementary Table 4), of which 14 are immune-related pathways listed in Table 3, and include NF-kappa B signaling pathway, NOD-like receptor signaling pathway, MAPK signaling pathway, and RIG-I receptor signaling pathway, and so on. Since the pathogen can directly induce immune response and cause disease in the host, we classified disease-related 
Table 2: Differentially expressed miRNAs (DEMs) in the whole body of B. belcheri challenged with pIC when compared with the negative control, as identified by deep sequencing. \# indicates conversed DEMs.

\begin{tabular}{|c|c|c|c|c|}
\hline Mature sequences (5'- 3') & miRNA id & $\begin{array}{c}\log 2 \text { (fold } \\
\text { change) }\end{array}$ & Regulation pattern & FDR \\
\hline uucgaaaucucucuguuccau & novel_mir27 & 8.40 & UP & $3.02 \mathrm{E}-97$ \\
\hline uaaccuguaaaucggauuugu & novel_mir183 & 8.10 & UP & $3.54 \mathrm{E}-82$ \\
\hline uguuaaauuaaugcuaugccac & novel_mir121 & 7.52 & UP & $1.01 \mathrm{E}-58$ \\
\hline uccuggguuggcuguuggcggcac & novel_mir196 & 7.25 & UP & $3.10 \mathrm{E}-50$ \\
\hline ucugggugucauccuccguagu & novel_mir33 & 6.89 & UP & $1.56 \mathrm{E}-40$ \\
\hline accecgacceggecuggcg & novel_mir95 & 6.04 & UP & $1.64 \mathrm{E}-24$ \\
\hline ucgcguaccgucugguuguggu & novel_mir59 & 5.83 & UP & $1.57 \mathrm{E}-21$ \\
\hline ucuucuauggcccgggaucuu & novel_mir35 & 5.56 & UP & $2.39 \mathrm{E}-18$ \\
\hline uuaggacauguucuacagcu & novel_mir157 & 5.56 & UP & $2.39 \mathrm{E}-18$ \\
\hline aacugggaccaugucuuccugc & novel_mir85 & 5.28 & UP & $1.51 \mathrm{E}-15$ \\
\hline aucauacaaaaggauuaccgagu & novel_mir116 & 5.09 & UP & $6.97 \mathrm{E}-14$ \\
\hline uuuagugaacuaugcaccaagu & novel_mir90 & 4.89 & UP & $2.38 \mathrm{E}-12$ \\
\hline ucagagaagccgagcugucaga & novel_mir156 & 4.23 & UP & $1.91 \mathrm{E}-08$ \\
\hline ucccccaguccggcggcaacgu & \#novel_mir136 & 4.13 & UP & $5.73 \mathrm{E}-08$ \\
\hline uuggccggccauggagggucc & novel_mir115 & 4.05 & UP & $1.17 \mathrm{E}-07$ \\
\hline ucgauggccggcugccuucucu & novel_mir81 & 3.81 & UP & $1.07 \mathrm{E}-06$ \\
\hline cgagucgucugauugcguuugc & novel_mir49 & 3.76 & UP & $1.54 \mathrm{E}-06$ \\
\hline gctgtgctgacaaactgccgtt & bbe-miR-31-3p & 3.66 & UP & $0.00 \mathrm{E}+00$ \\
\hline acgcagtgacgtcagcgcctct & bbe-miR-4856a-5p & 3.48 & UP & $0.00 \mathrm{E}+00$ \\
\hline agcgccgacggugguacacucucc & novel_mir38 & 3.39 & UP & $2.11 \mathrm{E}-05$ \\
\hline uuuggcacugguacuuuggagu & \#novel_mir65 & 3.20 & UP & $6.28 \mathrm{E}-05$ \\
\hline acuccucuggcauguuauuugu & novel_mir48 & 3.20 & UP & $6.28 \mathrm{E}-05$ \\
\hline aguaccgccagagagaccuuu & novel_mir147 & 3.16 & UP & $0.00 \mathrm{E}+00$ \\
\hline ucggagugagauuucaggguggcgg & novel_mir160 & 3.13 & UP & $9.07 \mathrm{E}-05$ \\
\hline caatgtaacagcagtgcagct & bbe-miR-33-2-3p & 2.76 & UP & $0.00 \mathrm{E}+00$ \\
\hline cccttatcacttcttccgccgag & bbe-miR-184-5p & 2.73 & UP & $1.27 \mathrm{E}-97$ \\
\hline aauccacagugauuuuggccaugu & novel_mir193 & 2.71 & UP & $5.64 \mathrm{E}-04$ \\
\hline aacaactaacatcactgccaag & bbe-miR-34b-3p & 2.71 & UP & $0.00 \mathrm{E}+00$ \\
\hline aggaagacauggucccaguugu & novel_mir50 & 2.67 & UP & $0.00 \mathrm{E}+00$ \\
\hline uuaugauuaucgagagaguucguu & novel_mir99 & 2.61 & UP & $8.03 \mathrm{E}-04$ \\
\hline taccctgtagatccggacttgt & bbe-miR-10c-5p & 2.52 & UP & $0.00 \mathrm{E}+00$ \\
\hline uaguguugcgacgaagaauagc & novel_mir36 & 2.43 & UP & $6.57 \mathrm{E}-23$ \\
\hline cgcctctgtttcgcgcgcaag & bbe-miR-210-3p & 2.24 & UP & $8.59 \mathrm{E}-76$ \\
\hline gtgattagctaaggtagctgata & bbe-miR-4890-5p & 2.20 & UP & $1.01 \mathrm{E}-05$ \\
\hline cttaccaggcagcatttagt & bbe-miR-200b-3p & 1.92 & UP & $0.00 \mathrm{E}+00$ \\
\hline ucuugggcauguagguuaugag & novel_mir150 & 1.79 & UP & $7.27 \mathrm{E}-201$ \\
\hline agucaccgucugucggcccguacga & novel_mir114 & 1.63 & UP & $7.60 \mathrm{E}-183$ \\
\hline uacuggccuccaaagucccggu & novel_mir10 & 1.58 & UP & $2.99 \mathrm{E}-19$ \\
\hline accaatatatcgaacatatgat & bbe-miR-190-3p & 1.55 & UP & $0.00 \mathrm{E}+00$ \\
\hline auacggggcaguuauauccaggu & novel_mir170 & 1.51 & UP & $2.42 \mathrm{E}-16$ \\
\hline
\end{tabular}




\begin{tabular}{|c|c|c|c|c|}
\hline uacggcuccccaagccgugguu & novel_mir31 & 1.47 & UP & $3.79 \mathrm{E}-21$ \\
\hline ctgtgcaacctgctagctctcc & bbe-let-7a-2-3p & 1.45 & UP & $0.00 \mathrm{E}+00$ \\
\hline gcagactggtgcttaacat & bbe-miR-252a-3p & 1.36 & UP & $0.00 \mathrm{E}+00$ \\
\hline uggcaguguauuuagcuggguaa & novel_mir52 & 1.30 & UP & $8.87 \mathrm{E}-28$ \\
\hline aucuggggcgguuaagguuauga & novel_mir26 & 1.16 & UP & $1.63 \mathrm{E}-35$ \\
\hline uggccuaaucccuguggauuu & novel_mir20 & 1.11 & UP & $1.58 \mathrm{E}-32$ \\
\hline tgcaacaatatttcatcagtgg & bbe-miR-2062-5p & 1.08 & UP & $0.00 \mathrm{E}+00$ \\
\hline ttctcgattgttctggtcca & bbe-miR-4887-5p & 1.05 & UP & $0.00 \mathrm{E}+00$ \\
\hline auucggguuuauaggaacauga & novel_mir19 & 1.04 & UP & 7.93E-49 \\
\hline gactgtacaacccgttacctt & bbe-let-7a-3p & 1.01 & UP & $0.00 \mathrm{E}+00$ \\
\hline acacuuauaggauuucucaccu & novel_mir166 & -1.09 & DOWN & $2.25 \mathrm{E}-26$ \\
\hline cagtaaatcacagtcttcctta & bbe-miR-7-3p & -1.17 & DOWN & $0.00 \mathrm{E}+00$ \\
\hline tgcagagcttgtgcgattggcg & bbe-miR-4888-5p & -1.23 & DOWN & $2.63 \mathrm{E}-35$ \\
\hline agacaacatcagaatgtccet & bbe-miR-4863-5p & -1.32 & DOWN & $1.82 \mathrm{E}-17$ \\
\hline agggacctgtagtcaacacgaca & bbe-miR-4869-3p & -1.33 & DOWN & $0.00 \mathrm{E}+00$ \\
\hline cacuggcccucuacgucuuga & novel_mir1 & -1.34 & DOWN & $0.00 \mathrm{E}+00$ \\
\hline tattgcacttatcctggectgt & bbe-miR-92d-3p & -1.40 & DOWN & $0.00 \mathrm{E}+00$ \\
\hline tgttcccaccttctgatgttgt & bbe-miR-4873-5p & -1.58 & DOWN & $0.00 \mathrm{E}+00$ \\
\hline tgcgtaggcgttgtgcacactgt & bbe-miR-242-5p & -1.59 & DOWN & $4.20 \mathrm{E}-48$ \\
\hline aggtctggatagttgcaatctt & bbe-miR-92b-5p & -1.66 & DOWN & $5.49 \mathrm{E}-11$ \\
\hline gcttagcagtgcaagtgtgac & bbe-miR-4866-3p & -2.04 & DOWN & $0.00 \mathrm{E}+00$ \\
\hline caucgacgccguccguuguagacgc & novel_mir130 & -2.12 & DOWN & $0.00 \mathrm{E}+00$ \\
\hline caatgtaactgcagtgcagcc & bbe-miR-33-3p & -2.16 & DOWN & $0.00 \mathrm{E}+00$ \\
\hline atgcggtgcggtggtagcaaccg & bbe-miR-2071-3p & -2.21 & DOWN & $0.00 \mathrm{E}+00$ \\
\hline gggcatgtgtgcatagggaaga & bbe-miR-2068-3p & -2.51 & DOWN & $0.00 \mathrm{E}+00$ \\
\hline gttcagcaccaaggacaggc & bbe-miR-2060c-3p & -2.60 & DOWN & $6.17 \mathrm{E}-47$ \\
\hline actggtgaaatgtagttgcgta & bbe-miR-2062-3p & -2.98 & DOWN & $0.00 \mathrm{E}+00$ \\
\hline uugggagucaggacaccauuguggu & novel_mir148 & -3.51 & DOWN & $8.24 \mathrm{E}-180$ \\
\hline cttggcacttgtggaattctctg & bbe-miR-182b-5p & -3.65 & DOWN & 7.92E-09 \\
\hline caucgacgccguccguuguggac & novel_mir131 & -3.80 & DOWN & $1.06 \mathrm{E}-40$ \\
\hline aattgcactagagtgatttgtt & bbe-miR-2076-5p & -3.90 & DOWN & $0.00 \mathrm{E}+00$ \\
\hline tttccacagcctctacacatgt & bbe-miR-2070-5p & -4.08 & DOWN & $0.00 \mathrm{E}+00$ \\
\hline ctaagtactagtgccgcaggtgt & bbe-miR-252a-5p & -4.64 & DOWN & $0.00 \mathrm{E}+00$ \\
\hline ugcucugagaugauaguaccagu & novel_mir194 & -6.65 & DOWN & $6.91 \mathrm{E}-10$ \\
\hline uucagaggacguaaacgcuuug & novel_mir140 & -6.89 & DOWN & $4.39 \mathrm{E}-11$ \\
\hline acuggguccagccggcucucccug & novel_mir158 & -7.19 & DOWN & $8.12 \mathrm{E}-13$ \\
\hline gccgagccggggcaggucgecccc & novel_mir138 & -8.30 & DOWN & $0.00 \mathrm{E}+00$ \\
\hline
\end{tabular}

KEGG terms along with immune-related pathways and found that many of them are involved in important virosis in vertebrates, such as viral carcinogenesis, hepatitis C, Influenza A, etc.

\section{Analysis of targeted genes in primarily immune- related KEGG pathway}

To further understand the regulatory roles of miRNAs in immune pathways under pIC challenge, 
targeted genes in predicted immune-related KEGG pathways were analyzed (Supplementary Table 5). We found that some miRNAs could regulate many typically immune-related targeted genes belonging to these immune pathways by being differentially expressed during the immune response. For example, bbe-miR$4856 \mathrm{a}-5 \mathrm{p}$ regulates the expression of genes encoding cytokine receptor domeless and interleukin 17D (IL17D) in the Jak-STAT signaling pathway; bbe-miR-210$3 p$ regulates genes encoding Ras-related protein Rab-7A (RAB7A) and NLR family CARD domain-containing protein 4 (NLRC4) in the Salmonella infection pathway; novel_mir49 and novel_mir38 regulate genes encoding TNF receptor-associated factor 4 (TRAF4) and tolllike receptor 1 (TLR1) respectively, in the Hepatitis C pathway; novel_mir49 can regulate TRAF2 and TRAF3, and nuclear factor NF-kappa-B p105 subunit (NFKB1) is a target gene of novel_mir50 in the NF-kappa B signaling pathway; novel_mir196 is a regulator of the gene encoding FAS-associated death domain protein (FADD) in the RIGI-like receptor signaling pathway, etc. Additionally, some target genes were shared by multiple pathways, such as TRAF3 and TRAF4, and NFKB1. Thus a miRNA could regulate multiple target genes and pathways at same time. Moreover, the same target gene was regulated by different miRNAs; for instance, the gene encoding Ras-related protein Rab-7A (RAB7A) was the target of bbe-miR4856a-5p as well as bbe-miR-210-3p in amphioxus under pIC challenge.

\section{DISCUSSION}

Innate immunity is the first line of defense against exogenous infection in all multicellular organisms. To date, only vertebrates have been demonstrated to possess adaptive immunity $[10,26]$. The original adaptive immunity could be traced to an early stage of vertebrate evolution, the jawless vertebrates such as cyclostomes, with more elaborate adaptive immune systems being developed in bony fish [27-29]. Thus, amphioxus must rely on the innate immune system in host defense against pathogen invasion due to the absence of adaptive immunity $[4,10]$. To avoid autoimmune damage, the immune responses must be precisely regulated by other non-coding molecules, such as miRNAs [30]. The role of miRNAs in regulation of immune responses has been well investigated in higher chordates, but related studies associated with basal chordates such as amphioxus are limited. Particularly, investigation of amphioxus miRNAs involved in immune responses primarily focus on experiments with bacteria/bacterial mimics challenge $[9,10,21]$, but miRNAs related to antiviral immune responses in amphioxus have yet not been characterized or analyzed. Here, we analyzed the genome-wide expression of miRNAs in $B$. belcheri treated by $\mathrm{pIC}$ and PBS (negative control) to identify antiviral responsive miRNAs using deep sequencing and speculate their regulatory role for target genes in immune-related pathways.

The results showed that the expression levels of two conserved miRNA sequences from a total of nine conserved miRNA sequences were significantly different in response to $\mathrm{pIC}$ challenge (fold-change $>3$ ). These two conserved miRNAs are homologous with bfl-miR-100$3 p$ and bfl-miR-4871-3p. Previous studies have found that down-regulation of miR-100 promoted a decrease in virus genome copies in shrimp and virus-infected shrimp mortality [31]. This inconsistency may be explained by amphioxus-specific (conserved between $B$. floridae and $B$. belcheri) miR-100 and different immune mechanisms between amphioxus and vertebrates. miRNAs homologous to bfl-miR-4871-3p were identified in B. belcheri infected with $V$. parahemolyticus, but significant expression of this miRNA was not detected between the treatment and control [10], indicating that amphioxus employs different miRNA molecules to regulate immune responses against virus and bacteria. In addition, analysis for target genes of three novel (belonging to B. belcheri-specific miRNAs) miRNAs with the most up-regulation after pIC challenge, predicted three genes (LOX2, RAR, GLYRI) associated with the metabolism of eicosanoids and retinoic acid, demethylation [32] to be the targets of novel_mir157, novel_mir183 and novel_mir121, respectively. Therefore, the highly up-regulated miRNAs in B. belcheri challenged by $\mathrm{pIC}$ negatively regulated part of genes are involved in metabolism and demethylation, perhaps to ensure adequate energy supply during immune responses by decreasing energy consumption in non-immune related biological processes. Interestingly, HSP70, NLR protein family, and $C 1 q L$ are important innate immune molecules in the defense response of host to virus invasion $[33,34]$. The most down-regulated molecules, novel-mir138, novelmir158 and novel-mir140 after pIC challenge promoted expression of key immune-related genes, indicating that these three novel miRNAs may regulate viral stress resistance by directly targeting immune-related genes.

33 known miRNAs with significant differential expression were identified in the treatment groups when compared with the negative control, indicating that these miRNAs participated in innate immune response in $B$. belcheri under pIC challenge. Based on sequence search in the miRBase database, some of these miRNAs were found to be conserved between vertebrates (such as Homo sapiens, hsa; Danio rerio, dre) and amphioxus, suggesting that they play fundamental roles in the immune system [9]. bbe-miR-92d-3p is an evolutionarily conserved molecule with hsa-miR-92a-3p, dre-miR-92a-3p, etc. Liu et al. found that miR-92a was down-regulated in swine testis cells infected with transmissible gastroenteritis virus (Coronaviridae family) [35]. Besides, using bioinformatics and hybrid PCR methods, Yang et al. detected reduced expression of bbe-miR-92d in B.belcheri infected with bacteria, and identified that miR-92d is a regulator for 
Table 3: List of the primarily immune-related KEGG pathways of target genes of differentially expressed miRNA (DEMs) between the $B$. belcheri control and that challenged with pIC.

\begin{tabular}{|l|c|c|c|}
\hline \multicolumn{1}{|c|}{ Pathway } & Pathway ID & $\boldsymbol{P}$-values & FDR \\
\hline NF-kappa B signaling pathway & ko04064 & $7.70 \mathrm{E}-05$ & $2.63 \mathrm{E}-03$ \\
\hline NOD-like receptor signaling pathway & ko04621 & $8.64 \mathrm{E}-05$ & $2.65 \mathrm{E}-03$ \\
\hline MAPK signaling pathway & ko04010 & $1.10 \mathrm{E}-04$ & $2.90 \mathrm{E}-03$ \\
\hline Endocytosis & ko04144 & $1.38 \mathrm{E}-04$ & $3.03 \mathrm{E}-03$ \\
\hline Chemokine signaling pathway & ko04062 & $1.97 \mathrm{E}-04$ & $3.78 \mathrm{E}-03$ \\
\hline Viral carcinogenesis & ko05203 & $2.11 \mathrm{E}-04$ & $3.81 \mathrm{E}-03$ \\
\hline Salmonella infection & ko05132 & $3.77 \mathrm{E}-04$ & $6.09 \mathrm{E}-03$ \\
\hline Hepatitis C & ko05160 & $5.90 \mathrm{E}-04$ & $8.23 \mathrm{E}-03$ \\
\hline Influenza A & ko05164 & $9.01 \mathrm{E}-04$ & $1.02 \mathrm{E}-02$ \\
\hline HTLV-I infection & ko05166 & $2.58 \mathrm{E}-03$ & $1.98 \mathrm{E}-02$ \\
\hline Jak-STAT signaling pathway & ko04630 & $3.84 \mathrm{E}-03$ & $2.64 \mathrm{E}-02$ \\
\hline Notch signaling pathway & ko04330 & $4.12 \mathrm{E}-03$ & $2.75 \mathrm{E}-02$ \\
\hline RIG-I-like receptor signaling pathway & ko04622 & $5.00 \mathrm{E}-03$ & $3.27 \mathrm{E}-02$ \\
\hline Viral myocarditis & ko05416 & $5.16 \mathrm{E}-03$ & $3.29 \mathrm{E}-02$ \\
\hline
\end{tabular}

the complement pathway via targeting of $\mathrm{C} 3$ [21]. In this study, we found up-regulation of bbe-miR-92d-3p after pIC challenge and the complement component $\mathrm{C} 3$ was predicted to be one of its target genes. This indicated that bbe-miR-92d-3p may be a regulator for acute immune response upon virus challenge by targeting $\mathrm{C} 3$ in the complement pathway. bbe-miR-10c-5p is well conserved with dre-miR-10c-5p and hsa-miR-10a-5p, etc., and belongs to the miR-10 family, which is highly conserved among different species [36]. Tu et al. demonstrated that miR-10 family plays a key role in suppressing proliferation and inducing apoptosis in humans, mouse and rat ovarian granulosa cells [36]. The up regulation of bbe-miR-10c-5p in our results suggested that apoptosis is an important biological process in antiviral immunity of amphioxus. bbe-miR-252a-5p and bbe-miR-242-5p are well conserved between amphioxus and insects, and deuterostomes (such as Saccoglossus kowalevskii, sko, Strongylocentrotus purpuratus, spu), suggesting that they are invertebrate-specific. Previous investigation reported that miR-252 suppressed the abundance of dengue virus transcript encoding envelope protein, and then decreased virus replication in Aedes albopictus [37]. The function of miR-242 has not been explored so far. Here, we revealed for the first time the immune response of miR242 to $\mathrm{pIC}$ challenge using deep sequencing in amphioxus. Notably, Liao et al. screened 49 significantly up-regulated and seven down-regulated miRNAs in the gill tissue of B.belcheri challenged by LPS after 12 post-injection (hpi) [9]. Jin et al. screened 14 significantly differentially expressed miRNAs in the whole body of B.belcheri infected with $V$. parahemolyticus [10]. In accordance with our results, 10 up-regulated miRNAs from Liao et al. research results and one (bbe-miR-210) reported by Jin et al. were included in the list of the up-regulated miRNAs in our results, respectively. Two miRNAs (bbe-miR-33 and bbe-miR-92d-3p) screened by Liao et al. and none reported by Jin et al. in down-regulated miRNA were found to overlap with down-regulated miRNAs in this study, respectively. However, we also found that the fold changes in expression compared to the control group were greater or less. This inconsistency may largely be caused by different immunogenic stimuli (bacterial mimics/ bacteria versus viral mimics).

The function of miRNAs appears to be that of gene regulation by targeting specific transcripts for inhibition of gene expression. Thus, functional analysis of the potential target mRNAs is essential for revealing miRNAmediated biological processes and pathways in antiviral immune response of amphioxus. In this study, the set of predicted target genes of DEMs was significantly enriched in different $\mathrm{GO}$ and $\mathrm{KEGG}$ terms. The GO terms having the most target genes are primarily involved in regulation, cell and single-organisms process. These observations are consistent with the results from immune response analysis of miRNAs to Cryptocryon irritans challenge in liver and gill of $L$. crocea [22]. Du et al. performed GO enrichment analysis of differentially expressed genes (DEGs) screened by transcriptome profiling of the spleen of Schizothorax prenanti challenged by $\mathrm{pIC}$ versus that of control. Three GO terms (related to immune response) obtained in our current study, namely immune system process, response to stimulus, and signaling, were among the $19 \mathrm{GO}$ terms from Du et al. [38]. Huang et al. reported that majority of the immune gene repertoire of amphioxus was well conserved and was shared with vertebrates [39]. Interestingly, despite 
a high functional overlap of potential target genes of DEMs between amphioxus and bony fish upon immune challenge, conserved DEMs with vertebrates account for a very low percentage (such as 9/197 novel miRNAs, 4.6\%). We inferred that the miRNA target site sequence in the 3' UTR of immune-related genes is more variable than the protein-coding regions, which may confer better evolutionary adaptation to formation of more complex regulatory networks. Certainly, this speculation needs to be further determined systematically by comparative genomic analysis.

At least 14 of the 42 significantly enriched pathways identified in this study were mainly immune-related. The results revealed some of the important immune pathways participating in the immune response of $B$. belcheri to pIC challenge. In addition, some pathways involved in wellknown human viral diseases, such as viral carcinogenesis, hepatitis C, influenza A, etc., were mainly detected in the list of immune-related KEGGs. Thus, amphioxus may be a potential model for investigating evolution of signaling pathways associated with human viral diseases. Subsequently, we found that miRNA has regulatory functions in antiviral immune response by targeting key genes involved in innate immune signaling pathways. Many of the target genes were found to encode cytokines such as IL-17D, cytokine receptor domeless (DOME) and interferon-induced helicase $\mathrm{C}$ domain-containing protein 1 (IFIH1). Previous studies have demonstrated that negative regulation of cytokines by increasing miRNA enhances interferon-mediated suppression of hepatitis B [40]. A subset of target genes encode pattern recognition receptors (PRRs) expressed by the innate immune cells to identify pathogen molecules, such as those in the TLR and NLR families. Changes in the abundance of these proteins could impact antiviral signaling [41], and hence the predicted regulatory function of miRNA for PRRs in amphioxus under virus invasion. Signal transducers and caspases involved in antiviral signaling, such as TRAF2, 3, 4, Caspase 8 (CASP8), were found to be encoded by our predicted target genes [42, 43]. Certainly, except for the above-mentioned, these target genes are involved in the innate immune system, and genes belonging to primarily immune-related pathways were also predicted in this study. These results indicated that known and newly identified DEMs are key regulators for important immune pathways by targeting PRRs, adaptors, cytokines and downstream signal transducers, etc., in antiviral immune response of B. belcheri. A part from these, a single miRNA can be a regulator of multiple target genes, while single target genes regulated by multiple miRNAs were also frequently detected here, suggesting the emergence of a complex and diverse miRNA-regulatory network in antiviral immune system in basal chordates.

In summary, deep-sequencing of the treatment and negative control groups was performed on three biological replicates, and the results were further confirmed by qRT-
PCR analysis. Moreover, we identified conserved and new miRNAs, and screened transcriptome-wide DEMs against viral mimics (pIC) in B. belcheri via deep sequencing and bioinformatics analysis. To explore the regulatory role of the miRNAs identified upon pIC challenge, we predicted and discussed the functions of the target genes of DEMs. Analysis of primarily immune-related target genes, their biological process and signaling pathways involved in antiviral immune response will deepen our understanding of antiviral immune function of posttranscriptional regulation of mRNAs in amphioxus. Additionally, miRNAs with acute immune responses will be preferential candidates for further functional analyses involving antiviral immunity of amphioxus. These results will provide useful information for investigating the miRNA evolution in antiviral immunity in vertebrates in future.

\section{MATERIALS AND METHODS}

\section{B.belcheri samples and viral mimics challenge}

Healthy B. belcheri adults were obtained from Beihai Marine Station of Nanjing University at Beihai (Guangxi Province, China), and were maintained as described earlier $[9,44]$. The amphioxus were acclimatized for about 6 days prior to challenge with pIC to empty the contents of the amphioxus bodies. Approximately 54 adult individuals were equally divided into six groups (9 amphioxiin each group). Next, $15 \mu \mathrm{l}$ pIC (dissolved in PBS, $100 \mu \mathrm{g} / \mathrm{ml}$ final concentration) was injected into the enterocoelia of each individual in the treatment group, after which they were carefully put back into filtered seawater. An equal dose of PBS was injected in the controls. Six individuals (three pIC treatments and three controls) were collected at each time-point, namely, 2, 6, and 12 hours post-injection (hpi) after pIC challenge. All samples (whole bodies of B.belcheri) were immediately frozen in liquid nitrogen for RNA extraction. Three biological replicates were used for each of the treated and negative control groups.

\section{RNA extraction, library construction and deep sequencing}

Total RNA was extracted from the whole body of each adult $B$. belcheri in the control and treatment groups using mirvanaTM miRNA isolation kit AM1560 (Ambion, USA) according to the manufacturer's protocol. We used Agilent 2100 Bioanalyzer (Agilent Technologies, USA) to detect RNA quality (RIN value) $\geq 7$ ) and estimate the concentration. Equal amount of total RNA from each individual (9 individuals, 3 amphixous at each timepoint) from treatment groups was pooled as treatment samples, and likewise from controls as control samples. 
Pooling of RNA samples at various time points after pIC challenge prior to sequencing may lead to bias in the results (e.g. a miRNA may first be up-regulated and down-regulated subsequently). However, we aimed at obtaining an overview of what occurs during immune challenge rather than understanding the dynamic changes in miRNA expression. Therefore, RNA sampling methods used in this study were similar to that in other experiments $[45,46]$. We generated three biological replicates each for the control and treated samples. Preparation of miRNA libraries was then performed according to the standard procedure at Beijing Genomics Institute (BGI, China). 1) RNA segments were separated for size using polypropylene acyl amine gel electrophoresis (PAGE), and 18-30nt longRNA molecules were selected and enriched; 2) 5-adenylated, 3-blocked single-stranded DNA adapters were linked to the 3'-termini of enriched small RNAs; 3) Reverse primer was hybridized to the 3 '-termini of RNA; 4) 5'-termini were linked to 5'adaptors; 5) cDNA was synthesized via reverse extension of the RT primer using Superscript II reverse transcriptase (Invitrogen, USA). 6) PCR amplification and cDNA enrichment was performed using both 3' and 5'adaptors. 7) cDNA libraries were purified by PAGE; 8) Quantitative analysis of the library was performed using Agilent 2100 Bioanalyzer (Agilent Technologies, USA). The prepared cDNA libraries were sequenced on the BGISEQ-500 platform (BGI, China; http://www.seq50.com/en/).

\section{Preprocessing analysis and identification of $B$. belcheri miRNA}

A total of six libraries were sequenced, with three biological replicates of treated and control samples each. The raw tags generated by deep sequencing were filtered by removing low-quality tags, tags with 5' primer contaminants and poly A stretches, tags without 3' primer and insertions, and tags of size shorter than $18 \mathrm{nt}$. After filtering, the length distribution of clean tags was analyzed, and clean tags were mapped to the $B$. belcheri genome (ftp://ttp.ncbi.nlm.nih.gov/genomes/, version: Haploidv18h27) using Bowtie2 with the following options: -q -L 16 -phred64 -p 6 [47]. Tags which matched perfectly were retained for downstream analysis. Non-coding small RNAs (sRNA), including rRNAs, snoRNAs, snRNAs, tRNAs, and other sRNA, were annotated by BLAST search in the NCBI GenBank and Rfam (http://rfam.sanger.ac.uk/) databases. After removal of repetitive sequences using RepeatMasker software (http://www.repeatmasker.org/), the remaining sRNAs tags were mapped to mRNA and were subsequently removed to avoid redundancy. The filtered tags were annotated by aligning to the $B$. belcheri miRNA database in miRBase 21.0 (http://www.mirbase.org/index.shtml) to obtain known miRNAs. The remaining non-annotated tags were used to predict novel miRNAs by mirDeep2 (https:// www.mdc-berlin.de/8551903/en/) with default options recommended by the software reports [48]. mirDeep2 is a suitable tool that can be used for discovery of miRNAs from deep sequencing data of animal clades, and can identify canonical and non-canonical miRNAs with an accuracy of $98.6-99.9 \%$ by a substantially improved algorithm [48, 49]. To obtain a rigorous result, the intersection of identified miRNAs in the three biological replicates was retained as the miRNA set of control or treated groups, and the secondary structures of novel miRNA precursor (pre-miRNAs) were analyzed using RNAfold (http://hackage.haskell.org/package/RNAFold). Furthermore, the predicted novel miRNA tags/sequences with mapped tags in less than 5 were discarded, and the remainder was retained as the final novel miRNA set. The novel miRNAs were annotated by searching in the animal database of miRBase21.0, allowing for a maximum of two mismatches, and those annotated to known miRNAs of other animals were considered to be conserved.

\section{Identification of differentially expressed miRNA under pIC challenge}

The expression levels of all sRNAs in the six libraries were normalized by TPM values: absolute tag count multiply $10^{6} /$ total count of clean tags [22]. We evaluated repeatability among the three biological replicates by pairwise comparison of the samples based on TPM values, and calculated Pearson's correlations of each pair. Some miRNAs were expressed in only one of control and treated groups, but not in another, indicating that expression of these miRNAs was related to pIC challenge. To avoid the omitting these miRNAs, the union of identified miRNAs in control and treated groups was taken as the final list of miRNAs for analysis. DEMs between the control and treated groups (treatment vs. control) were screened using DEGseq software, which is an $\mathrm{R}$ program suitable for different expression analysis of deep sequencing in biological replicates [50]. miRNAs with fold change $(\mathrm{FC}) \geq 2\left(\mid \log _{2}\right.$ ratio $\left.\mid \geq 1\right)$ and $P$-values corrected by the false discovery rate $(\mathrm{FDR})<0.001$ were considered to be significantly differentially expressed.

\section{Target gene prediction and functional enrichment analysis of DEMs}

To understand the regulatory function of DEMs in the immune response of $B$. belcheri to pIC challenge, the potential target genes of DEMs were predicted using two approaches: RNAhybrid (https://bibiserv.cebitec.unibielefeld.de/download/tools/rnahybrid.html), and miRanda (http://www.microrna.org/microrna/getDownloads.do). The intersection of the two prediction results was then taken as the reliable set of target genes of DEMs for 
further analysis.

To further explore the functions of target genes that were regulated by DEMs in anti-pIC immunity in biological processes and signaling pathways, all proteincoding genes were first annotated against the GO database (http://www.geneontology.org/), non-redundant (NR), UniProt (http://www.uniprot.org/), and KEGG (http:// www.genome.jp/kegg) databases. The GO enrichment analysis for the target genes of the DEMs was performed by Fisher's exact test ( $P$-values) in Blast2GO pipeline [51], and $P$-values were used to conduct multiple test correction by FDR. GO terms with FDR $<0.05$ were considered to be significantly enriched. Moreover, we reduced the redundant terms in the list of significantly enriched GO terms using GO trimming tool (http://lucy. ceh.uvic.ca/go_trimming/cbr_go_trimming.py). KEGG enrichment analysis of target genes of miRNAs was implemented in KOBAS 2.0 software [52], and terms with significantly enriched values $($ FDR value $<0.05$ ) were retained.

\section{Validation of miRNA deep sequencing data}

For validation of the miRNA deep sequencing results, 10 DEMs (five up- and five down-regulated) were randomly selected, and a portion of the RNA preparations used in miRNA deep sequencing was used for qRTPCR analysis. Total RNA was treated by RNase-free DNase (Qiagen, Germany) to remove any residual DNA contamination. Reverse transcription of total RNA was performed using TransScript Green miRNA Two-Step qRT-PCR SuperMix (TransGenBiotech, China) according to the recommendations in the product manual. We used TransStart TipGreen qPCR SuperMix (TransGen Biotech, China) to conduct qRT-PCR analysis on an ABI 7300 Realtime PCR system (Applied Biosystems, USA) following reaction conditions suggested by TransGenBiotech. All reactions were analyzed in triplicate. The relative quantification of each miRNA was calculated based on the $2^{-\triangle \Delta C T}$ method [53]. All primers are listed in Supplementary Table 6. A U6 gene was selected as the internal control. IBM SPSS Statistics 22 was employed for statistical analysis.

\section{ACKNOWLEDGMENTS}

This work was supported by the 973 project of Ministry of Science and Technology of China (Grants No. 2013CB835300) and the Natural Science Foundation of China (41272008).

\section{CONFLICTS OF INTEREST}

The authors declare that they have no conflicts of interest.

\section{DATA ACCESSIBILITY}

The data supporting the results of this article have been submitted to NCBI Sequence Read Archive (SRA) repository [Experiment accession numbers: SRX2992234].

\section{REFERENCES}

1. Satoh N, Rokhsar D, Nishikawa T. Chordate evolution and the three-phylum system. Proc Biol Sci. 2014; 281:20141729.

2. Putnam NH, Butts T, Ferrier DE, Furlong RF, Hellsten U, Kawashima T, Robinson-Rechavi M, Shoguchi E, Terry A, Yu JK, Benito-Gutiérrez EL, Dubchak I, Garcia-Fernàndez $\mathrm{J}$, et al. The amphioxus genome and the evolution of the chordate karyotype. Nature. 2008; 453:1064-1071.

3. Wang L, Su H, Feng P, Zhang S. Amphioxus in comparative genomics and molecular developmental biology. Transaction of Oceanology \& Limnology. 2005:84-91.

4. Yue JX, Yu JK, Putnam NH, Holland LZ. The transcriptome of an amphioxus, Asymmetron lucayanum, from the Bahamas: A window into chordate evolution. Genome Biol Evol. 2014; 6:2681-2696.

5. Wang Y, Zhang S. EF1 $\alpha$ is a useful internal reference for studies of gene expression regulation in amphioxus Branchiostoma japonicum. Fish Shellfish Immunol. 2012; 32:1068-1073.

6. Lei M, Liu H, Liu S, Zhang Y, Zhang S. Identification and functional characterization of viperin of amphioxus Branchiostoma japonicum: Implications for ancient origin of viperin-mediated antiviral response. Dev Comp Immunol. 2015; 53:293-302.

7. Liu S, Liu Y, Yang S, Huang Y, Qin Q, Zhang S. Evolutionary conservation of molecular structure and antiviral function of a viral receptor, LGP2, in amphioxus Branchiostoma japonicum. Eur J Immunol. 2015; 45:34043416.

8. Liu Z, Li L, Li H, Zhang S, Ji G, Sun Y. EST analysis of the immune-relevant genes in Chinese amphioxus challenged with lipopolysaccharide. Fish Shellfish Immunol. 2009; 26:843-849.

9. Liao X, Yang L, Zhang Q, Chen J. microRNA expression changes after lipopolysaccharide treatment in gills of amphioxus Branchiostoma belcheri. Dev Comp Immunol. 2017; 70:39-44.

10. Jin P, Li S, Sun L, Lv C, Ma F. Transcriptome-wide analysis of microRNAs in Branchiostoma belcheri upon Vibrio parahemolyticus infection. Dev Comp Immunol. 2017; 74:243-252.

11. Ambros V, Bartel B, Bartel DP, Burge CB, Carrington JC, Chen X, Dreyfuss G, Eddy SR, Griffiths-Jones S, Marshall M, Matzke M, Ruvkun G, Tuschl T. A uniform system for microRNA annotation. RNA. 2003; 9:277-279.

12. Bagga S, Bracht J, Hunter S, Massirer K, Holtz J, Eachus 
R, Pasquinelli AE. Regulation by let-7 and lin-4 miRNAs results in target mRNA degradation. Cell. 2005; 122:553563.

13. Lim LP, Lau NC, Garrettengele P, Grimson A, Schelter JM, Castle J, Bartel DP, Linsley PS, Johnson JM. Microarray analysis shows that some microRNAs downregulate large numbers of target mRNAs. Nature. 2005; 433:769-773.

14. Selbach M, Schwanhausser B, Thierfelder N, Fang Z, Khanin R, Rajewsky N. Widespread changes in protein synthesis induced by microRNAs. Nature. 2008; 455:58-63.

15. Gommans WM, Berezikov E. Controlling miRNA regulation in disease. Methods Mol Biol. 2012; 822:1-18.

16. O'Connell RM, Rao DS, Chaudhuri AA, Baltimore D. Physiological and pathological roles for microRNAs in the immune system. Nat Rev Immunol. 2010; 10:111-22.

17. O'Connell RM, Rao DS, Baltimore D. microRNA regulation of inflammatory responses. Annu Rev Immunol. 2012; 30:295-312.

18. Baltimore D, Boldin MP, O'Connell RM, Rao DS, Taganov KD. MicroRNAs: new regulators of immune cell development and function. Nat Immunol. 2008; 9:839-845.

19. Tsitsiou E, Lindsay MA. microRNAs and the immune response. Curr Opin Pharmacol. 2009; 9:514-520.

20. Lu J, Getz G, Miska EA, Alvarez-Saavedra E, Lamb J, Peck D, Sweet-Cordero A, Ebert BL, Mak RH, Ferrando AA, Downing JR, Jacks T, Horvitz HR, et al. MicroRNA expression profiles classify human cancers. Nature. 2005; 435:834-838.

21. Yang R, Zheng T, Cai X, Yu Y, Yu C, Guo L, Huang S, Zhu W, Zhu R, Yan Q, Ren Z, Chen S, Xu A. Genomewide analyses of amphioxus microRNAs reveal an immune regulation via miR-92d targeting C3. J Immunol. 2013; 190:1491-1500.

22. Qiao Y, Mao Y, Wang J, Chen R, Su YQ, Chen J, Zheng WQ. Analysis of liver and gill miRNAs of Larimichthys crocea against Cryptocryon irritans challenge. Fish Shellfish Immunol. 2016; 59:484-491.

23. Andreassen R, Woldemariam NT, Egeland IØ, Agafonov $\mathrm{O}$, Sindre H, Høyheim B. Identification of differentially expressed Atlantic salmon miRNAs responding to salmonid alphavirus (SAV) infection. BMC Genomics. 2017; 18:349.

24. Jagadeeswaran G, Zheng Y, Sumathipala N, Jiang H, Arrese EL, Soulages JL, Zhang W, Sunkar R. Deep sequencing of small RNA libraries reveals dynamic regulation of conserved and novel microRNAs and microRNA-stars during silkworm development. BMC Genomics. 2010; 11:52.

25. Huang $\mathrm{S}$, Chen $\mathrm{Z}$, Yan $\mathrm{X}, \mathrm{Yu} \mathrm{T}$, Huang G, Yan Q, Pontarotti PA, Zhao H, Li J, Yang P, Wang R, Li R, Tao X, et al. Decelerated genome evolution in modern vertebrates revealed by analysis of multiple lancelet genomes. Nat Commun. 2014; 5:5896-5896.

26. Hoebe K, Janssen E, Beutler B. The interface between innate and adaptive immunity. Nat Immunol. 2004; 5:971-
974.

27. Venkatesh B, Lee AP, Ravi V, Maurya AK, Lian MM, Swann JB, Ohta Y, Flajnik MF, Sutoh Y, Kasahara M, Hoon S, Gangu V, Roy SW, et al. Elephant shark genome provides unique insights into gnathostome evolution. Nature. 2014; 505:174-179.

28. Han BW, Herrin BR, Cooper MD, Wilson IA. Antigen recognition by variable lymphocyte receptors. Science. 2008; 321:1834-37.

29. Nagawa F, Kishishita N, Shimizu K, Hirose S, Miyoshi M, Nezu J, Nishimura T, Nishizumi H, Takahashi Y, Hashimoto S, Takeuchi M, Miyajima A, Takemori T, et al. Antigen-receptor genes of the agnathan lamprey are assembled by a process involving copy choice. Nat Immunol. 2007; 8:206-213.

30. Qu Z, Li W, Fu B. MicroRNAs in autoimmune diseases. Biomed Res Internat. 2014; 2014:527895.

31. Yang L, Yang G, Zhang X. The miR-100-mediated pathway regulates apoptosis against virus infection in shrimp. Fish Shellfish Immunol. 2014; 40:146-153.

32. Fang R, Chen F, Dong Z, Hu D, Barbera AJ, Clark EA, Fang J, Yang Y, Mei P, Rutenberg M, Li Z, Zhang Y, Xu Y, et al. LSD2/KDM1B and its cofactor NPAC/GLYR1 endow a structural and molecular model for regulation of $\mathrm{H} 3 \mathrm{~K} 4$ demethylation. Mol Cell. 2013; 49:558-570.

33. Oglesbee MJ, Pratt M, Carsillo T. Role for heat shock proteins in the immune response to measles virus infection. Viral Immunol. 2002; 15:399-416.

34. Wilmanski JM, Petnicki-Ocwieja T, Kobayashi KS. NLR proteins: integral members of innate immunity and mediators of inflammatory diseases. J Leukoc Biol. 2008; 83:13-30.

35. Liu X, Zhu L, Liao S, Xu Z, Zhou Y. The porcine microRNA transcriptome response to transmissible gastroenteritis virus infection. PLoS One. 2015; 10:e120377.

36. Jiajie T, Yanzhou Y, Hoi-Hung AC, Zi-Jiang C, Wai-Yee C. Conserved miR-10 family represses proliferation and induces apoptosis in ovarian granulosa cells. Sci Rep. 2017; 7:41304.

37. Yan H, Zhou Y, Liu Y, Deng Y, Chen X. miR-252 of the Asian tiger mosquito Aedes albopictus regulates dengue virus replication by suppressing the expression of the dengue virus envelope protein. J Med Virol. 2013; 86:14281436.

38. Du X, Li Y, Li D, Lian F, Yang S, Wu J, Liu H, Bu G, Meng F, Cao X, Zeng X, Zhang H, Chen Z. Transcriptome profiling of spleen provides insights into the antiviral mechanism in Schizothorax prenanti after poly (I: C) challenge. Fish Shellfish Immunol. 2017; 62:13-23.

39. Huang S, Yuan S, Guo L, Yu Y, Li J, Wu T, Liu T, Yang M, Wu K, Liu H, Ge J, Yu Y, Huang H, et al. Genomic analysis of the immune gene repertoire of amphioxus reveals extraordinary innate complexity and diversity. Genome Res. 2008; 18:1112-1126. 
40. Gao D, Zhai A, Qian J, Li A, Li Y, Song W, Zhao H, Yu X, Wu J, Zhang Q, Kao W, Wei L, Zhang F, Zhong Z. Downregulation of suppressor of cytokine signaling 3 by miR122 enhances interferon-mediated suppression of hepatitis B virus. Antiviral Res. 2015; 118:20-28.

41. Kawai T, Akira S. Antiviral signaling through pattern recognition receptors. J Biochem. 2007; 141:137-45.

42. Zhao K, Zhang M, Zhang L, Wang P, Song G, Liu B, Wu H, Yin Z, Gao C. Intracellular osteopontin stabilizes TRAF3 to positively regulate innate antiviral response. Sci Rep. 2016; 6:23771.

43. El Maadidi S, Faletti L, Berg B, Wenzl C, Wieland K, Chen ZJ, Maurer U, Borner C. A novel mitochondrial MAVS/ Caspase-8 platform links RNA virus-induced innate antiviral signaling to Bax/Bak-independent apoptosis. J Immunol. 2014; 192:1171-1183.

44. Zhang QL, Zhu QH, Liao X, Wang XQ, Chen T, Xu HT, Wang J, Yuan ML, Chen JY. Selection of reliable reference genes for normalization of quantitative RT-PCR from different developmental stages and tissues in amphioxus. Sci Rep. 2016; 6:37549.

45. Liu S, Chen G, Xu H, Zou W, Yan W, Wang Q, Deng H, Zhang H, Yu G, He J, Weng S. Transcriptome analysis of mud crab (Scylla paramamosain) gills in response to Mud crab reovirus (MCRV). Fish Shellfish Immunol. 2017; 60:545-553.

46. Etebari K, Palfreyman RW, Schlipalius D, Nielsen LK, Glatz RV, Asgari S. Deep sequencing-based transcriptome analysis of Plutella xylostella larvae parasitized by Diadegma semiclausum. BMC Genomics. 2011; 12:446.
47. Langmead B, Trapnell C, Pop M, Salzberg SL. Ultrafast and memory-efficient alignment of short DNA sequences to the human genome. Genome Biol. 2009; 10:R25.

48. Friedländer MR, Mackowiak SD, Li N, Chen W, Rajewsky N. miRDeep2 accurately identifies known and hundreds of novel microRNA genes in seven animal clades. Nucleic Acids Res. 2012; 40:37-52.

49. Friedländer MR, Chen W, Adamidi C, Maaskola J, Einspanier R, Knespel S, Rajewsky N. Discovering microRNAs from deep sequencing data using miRDeep. Nat Biotechnol. 2008; 26:407-415.

50. Wang L, Feng Z, Wang X, Zhang X. DEGseq: an R package for identifying differentially expressed genes from RNAseq data. Bioinformatics. 2010; 26:136-138.

51. Conesa A, Götz S, Garcíagómez JM, Terol J, Talón M, Robles M. Blast2GO: a universal tool for annotation, visualization and analysis in functional genomics research. Bioinformatics. 2005; 21:3674-6.

52. Xie C, Mao X, Huang J, Ding Y, Wu J, Dong S, Kong L, Gao G, Li CY, Wei L. KOBAS 2.0: a web server for annotation and identification of enriched pathways and diseases. Nucleic Acids Res. 2011; 39:W316-22.

53. Livak KJ, Schmittgen TD. Analysis of relative gene expression data using real-time quantitative PCR and the 2- $\Delta \Delta$ CT method. Methods. 2001; 25:402-408. 